\title{
Le rythme étranger et la catalyse ponctuelle de la culture. Dialogues possibles entre Barthes et
}

\section{Lotman}

\section{Pierluigi Basso Fossali}

\section{Qrevues.org}

Édition électronique

URL : http://signata.revues.org/1160

DOI : 10.4000/signata. 1160

ISSN : 2565-7097

\section{Éditeur \\ Presses universitaires de Liège (PULg)}

\section{Édition imprimée}

Date de publication : 31 décembre 2015

Pagination : 447-462

ISBN : 978-2-87562-087-3

ISSN : 2032-9806

Ce document vous est offert par

Bibliothèque Diderot de Lyon

UNR-RA $=$ It

Référence électronique

Pierluigi Basso Fossali, « Le rythme étranger et la catalyse ponctuelle de la culture. Dialogues possibles entre Barthes et Lotman », Signata [En ligne], 6 | 2015, mis en ligne le 31 décembre 2016, consulté le 10 novembre 2017. URL : http://signata.revues.org/1160; DOI : 10.4000/signata.1160 


\title{
VARIA
}

\section{Le rythme étranger et la catalyse ponctuelle de la culture. Dialogues possibles entre Barthes et Lotman}

\author{
Pierluigi Basso Fossali \\ Université Lyon 2 / ICAR, ENS de Lyon
}

\section{Face à l'héritage hjelmslevien : texte et histoire ${ }^{1}$}

Pour Barthes tout comme pour Lotman², les années 1960 s'ouvrent par une confrontation discrète avec la sémiotique hjelmslevienne; pour Barthes (1960a; 1960b), il y a avant tout la question de la recherche des unités minimales dans la mode et dans le cinéma : toutefois, Hjelmslev est cité de manière latérale jusqu'aux Éléments de sémiologie qui datent de 1965. La saison hjelmslevienne de Barthes est marquée par une adhésion à une posture méthodologique et à une série de concepts opératoires, capables d'éclaircir la forme d'organisation du langage. En revanche, Barthes n'a jamais adopté l'épistémologie hjelmslevienne.

Après la parution controversée du Système de la mode, il commence à souligner de plus en plus que la réflexion sur la connotation était simplement l'occasion de rabattre le "prestige de la linguistique, qui, jusqu'à ces jours, a réduit le langage à la phrase et à ses composantes lexicales et syntaxiques ", ce qui infléchit toute

1. J'ai le plaisir de remercier Odile Le Guern et François Provenzano pour la relecture précieuse de cet article et Anne-Marie Houdebine, Kalevi Kull et Bernard Paqueteau pour avoir sollicité de manière indirecte ou directe ce dialogue entre la sémiologie barthésienne et la théorie lotmanienne.

2. Selon Zenkine (2010, p. 84), Lotman se disait «sceptique " quant à «la valeur proprement scientifique » des écrits de Barthes, et à son tour, ce dernier n'a jamais cité le sémiologue russe. Notre tentative est de trouver des intersections productives entre la pensée lotmanienne et les contributions barthésiennes à la sémiotique, au-delà des actes manqués et des méfiances avouées en privé. 
la pensée occidentale classique qui estime pouvoir « disposer tous les sens d'un texte en cercle autour du foyer de la dénotation (le foyer : centre, gardien, refuge, lumière de la vérité » (Barthes, 1970b, p. 124).

Au début des années 1970, Hjelmslev est déjà devenu, aux yeux de Barthes, un disciple de la grammatica universalis des Modistes, chez qui la diversité des grammaires culturelles est sacrifiée au profit de la reconnaissance de la centralité des relations entre les signes. Le problème est qu'il faudrait pousser la généralisation non pas vers la reconnaissance d'une langue abstraite, mais vers un paradigme praxique de l'" intersigne ", ce qui veut dire que Barthes a déjà abandonné la fascination pour la structure avec la vision plus dynamique et fuyante de la structuration (Barthes, 1970c, p. 551). L'abolition d'un «fond» dénotatif comme paramètre de la signification ne peut pas être substituée par un autre fond stable : la forme de la langue. Barthes fait encore référence à Hjelmslev pour le primat de la forme mais pour affirmer «le recul infini des signifiés $»^{3}$ (Barthes, 1970d, p. 639). Dans cette perspective, Barthes déplace son centre d'intérêt, des codes, avec l'idée de pouvoir pénétrer ainsi l'idéologie cachée dans le langage, aux textes, où les enjeux culturels sont encore vifs et dynamiques.

De son côté, Lotman a fait référence explicitement à la leçon hjelmslevienne afin de problématiser la notion de texte: dans le paragraphe "Structures textuelles et extratextuelles » de ces Leçons sur la poétique structurale (Lotman, 1964) l'exigence d'une textologie, réclamée par Lichacev (1962), trouve son point de repère immédiat dans la contribution de Hjelmslev et de ses Prolégomènes, publiés en russe en 1960, sur Novoe v Lingvistike («Le nouveau en linguistique»). Ce qui frappe Lotman, c'est l'aptitude hjelmslevienne à assimiler le texte avec la dimension discursive globale de la parole qui, bien qu'attestée, n'a pas des frontières certaines et est susceptible de se reproduire à l'infini. Mais, le deuxième problème, aux yeux de Lotman, est que cette syntagmatique infinie et inanalysée devrait être remplacée par une reconstruction procédurale, vu que la langue contiendrait déjà, sur le plan des combinaisons abstraites, toutes les réalisations textuelles effectives. Au-delà de certaines simplifications de la pensée hjelmslevienne, Lotman est très lucide dans la critique d'une perspective textologique qui renonce à caractériser le statut d'objet de l'œuvre artistique, avec ses supports et ses finalités communicatives spécifiques.

Le problème n'est pas la distinction entre une analyse générale, consacrée à la langue, et une analyse particulière, concentrée sur la parole, mais la préservation de l'histoire du texte, de son projet de réalisation aux interprétations attestées. Bref, Lotman cherche une conciliation entre la reconnaissance sémiologique d'une structure d'organisation du texte, toujours objectivable, et la vision culturaliste de Boris Tomaševskij, où l'œuvre d'art est saisie comme un événement changeant, dynamique (Lotman, 1964, trad. it. p. 88).

On peut entrevoir ici un premier point de contact avec Barthes, dans son idée de passer de la structure à la structuration. Le fait que le texte est toujours un objet

3. La même idée est exprimée littéralement dans Barthes (1972, p. 181). 
qui entre dans des processus de communication est à la base de l'intervention programmatique "La linguistique du discours " prononcée à Kazimierz en 1966, où eut lieu la fondation de l'Association Internationale de Sémiotique. Le discours est pour Barthes «toute étendue finie de parole, unifiée du point de vue du contenu, émise et structurée à des fins de communication secondaires, culturalisée par des facteurs autres que ceux de la langue " (Barthes, 1966d, p. 612). Tout au long de cette intervention, le mot-clé de Barthes est intégration (ibid., p. 614-616) : il y a un rapport d' "intégration" entre la linguistique et la translinguistique, entre le texte et l'extratextuel, opérant ainsi le passage entre la phrase et l'" au-delà de la phrase » promu, selon Barthes, par « les formalistes russes, Jakobson, Shscheglov et ses collègues soviétiques» (ibid., p. 613).

Pour Lotman, le texte a bien une " identité graphique », une syntaxe d'épellation spécifique, mais il peut survivre à ses états de manifestations fragmentaires, aux contingences de la transmission culturelle, ce qui montre qu'il ne peut pas se préserver dans le temps sans son ancrage historique et son statut social.

De telles réflexions anticipent la théorie de Nelson Goodman (1968) et signalent aussi, sur un plan plus technique, le caractère toujours elliptique du texte : par exemple, les points de suspension ou les omissions des strophes chez Pouchkine. Se pose alors le problème de l'extratextualité. Le raisonnement est simple : si la vision structuraliste saisit le texte comme un réseau de rapports, la même perspective doit être gardée entre le texte et son entour culturel (Lotman, 1964, tr. it. p. 90).

La vision hjelmslevienne du texte est alors problématisée à travers deux passages : (i) la négation de l'indifférence du plan de l'expression par rapport à la manifestation des contenus (le signifiant est toujours un interprétant du texte); (ii) la non-autonomie du texte, qui n'est pas le «niveau supérieur » de la signification discursive (ibid., p. 93) par rapport aux choix linguistiques et stylistiques (les « artifices»).

D’après Lotman, la valeur de la structure poétique de l'œuvre, par exemple l'organisation rythmique, relève de l'histoire d'une littérature nationale spécifique. Sa traduction dans une autre langue ne pose pas seulement le problème du respect de son organisation textuelle interne, mais il faut faire référence aussi au rôle qu'elle joue dans son milieu social afin de trouver une structure poétique dans la langue d'arrivée, qui interprète une fonction similaire dans sa propre culture d'origine (par exemple, la reprise nostalgique d'un classicisme ancien).

Bien évidemment, on pourrait souligner la présence, dans la pensée de Hjelmslev, du sub-logique, des normes, des appréciations collectives. Or, sans s'attarder sur les aspects philologiques controversés de la réception des Prolégomènes de la part de Lotman et Barthes, il faut se concentrer aujourd'hui sur leur commune réaction : la nécessité de passer de la structure à la structuration, des déterminations sémantiques délimitées par les lisières textuelles aux dynamiques historiques de production de sens. 


\section{La catalyse : du paradigme à l'environnement}

\subsection{Le zéro-problème}

Pour Barthes, la lecture n'est qu'une structuration:

Je ne suis pas le seul à opposer ainsi la structure et la structuration. Cette opposition s'inscrit dans le jeu historique de la sémiologie littéraire. Il s'agit, en effet, de dépasser le statisme de la première sémiologie, qui essayait précisément de retrouver des structures, des structures-produits, des espaces d'objets dans un texte, pour retrouver ce que Julia Kristeva appelle une productivité, c'est-àdire un travail, une prise, un branchement sur l'infini permutatoire du langage. Il s'agit d'évaluer exactement le degré de clôture d'un texte (Barthes, 1970a, p. 659).

L' "intersens" (ibid., p. 660) de Barthes n'est pas seulement une intersémantique entre les textes mais il relève aussi des espaces paradigmatiques de la langue. La présence forte de l'organisation paradigmatique chez Barthes et dans la Structure du texte poétique de Lotman (1970), montre qu'elle est conçue comme une extension possible de la signification, comme s'il y avait une résonance du dit avec toutes les voix énonciatives actualisables ou traductibles du dire, une sorte d'espace sémantique contrastif par rapport au parcours linéaire univoque des combinaisons.

Dans le paragraphe « Le concept de texte» (Lotman, 1970, tr. fr. p. 89), Lotman indique les rôles du paradigme : l'usage d'un rythme a une signification qui relève des systèmes alternatifs présents dans une certaine culture historique, et qui assume, bien évidemment, une valeur différente là où ce rythme est la seule solution admise. En particulier, Lotman semble enclin à généraliser le fait que chaque production textuelle n'est qu'une manifestation des «structures inachevées" (ibid., p. 90), ce qui va présentifier l'absence de certains paradigmes. L'absence de manifestation intégrale est une " grandeur pleinement réelle et mesurable » de la communication (ibid.) par l'articulation entre la textualité et le système d'attentes des lecteurs, lié à son tour au cadre linguistique et pragmatique.

C'est exactement à ce propos que Lotman fait une citation explicite au travail de Barthes : il faut reconnaître que la structure inachevée, «incomplète » (ibid., p. 90), relève d'un problème plus général, c'est-à-dire du « rôle constructif du zéro signifiant (zéro-problème - en français dans le texte), du signifié sémantique de la pause » (ibid.). Dans la note en bas de page, il y a un renvoi, même s'il est incorrect, à «M. (sic, Henri) Frei ${ }^{4}$ », mais à côté de cette lecture, probablement indirecte - il n'y a pas le titre de l'article de Frei mais seulement la date : 1953 -, on trouve Le degré zéro de l'écriture de la même année (Barthes, 1953, p. 217). Lotman cite en particulier le chapitre «L'écriture et le silence » du livre de Barthes, en témoignant d'une disponibilité à faire référence au sémiologue de Cherbourg qu'on peut retrouver aussi dans « À propos de Khlestakov» (1975b), lorsque Lotman fait un

4. Frei (1950). La référence de Lotman est en réalité une intervention de Frei parue en 1953 dans les Cahiers Ferdinand de Saussure. 
renvoi aux Mythologies (Barthes, 1957) pour les diverses orientations sociales dans la consommation d'alcool ${ }^{5}$.

Nous n'avons pas le temps ici de thématiser cette question épineuse, liée à la tradition saussurienne; nous pouvons la synthétiser en disant que le zéro-problème est un signe implicite et oppositionnel, certes paradigmatique mais encore blanc, neutre, dépourvu de manifestation ${ }^{6}$. Afin de combler le vide - le zéro-problème -, il faut opérer une catalyse, laquelle n'est pas limitée aux lisières textuelles, mais prend en charge les structures paradigmatiques en résonance avec le zéro signifiant, aussi bien que des «éléments extra-systématiques " (Lotman, 1970, trad. fr. p. 91). Il faut rappeler que dans la première page de La culture et l'explosion, on trouve encore, après 30 ans, le même renvoi au signifiant zéro, au "zéro significatif », à cette expression d'une absence (Lotman, 1993, tr. fr. p. 21, n. 1). C'est dans ce livre que Lotman met au centre de la logique de l'explosion les catalyseurs. D'ailleurs, même pour Barthes, la question du Signifiant zéro a résisté dans toute son actualité jusqu'aux réflexions sur Le neutre de 1977-1978 (Barthes, 1978, p. 54).

\subsection{La catalyse et l'inachèvement textuel}

La catalyse est une notion hjelmslevienne qui renvoie à la nécessité d'expliciter des éléments elliptiques à travers des cadres de référence ou par des présuppositions logiques et narratives (cf. Greimas \& Courtés, 1979, p. 33). Toutefois, il est mieux de préciser deux aspects de cette notion. Le premier relève de l'origine étymologique et disciplinaire du terme : le mot veut dire kata « dessous » et lysis « dissolution", "détachement", " dégagement». On peut alors bien comprendre la notion chimique correspondante qui pense la catalyse comme le dégagement d'un processus sous-tendu par un élément activateur qui n'est pas nécessairement modifié par la transformation réalisée. On souligne en particulier qu'il y a l'accélération d'une réaction ou la réorientation de sa dynamique.

Dans la vision lotmanienne, les phénomènes textuels d'ellipse - où la manifestation incomplète des structures construit l'attente pour un élément extratextuel qui pourrait remplacer les traits manquants - sont les aspects les plus importants à détecter pour rendre compte de la dynamis culturelle. Il est plutôt facile de voir dans cette perspective lotmanienne une référence au phénomène de la catalyse, évoqué indirectement par le zéro-problème et qui sera explicité dans les années 1980. Il faut remarquer surtout, que, d'après Lotman, la manifestation "vide" est avant tout un facteur de ralentissement de la lecture, normalement recherchée par les stratégies artistiques, et ensuite un facteur de forte accélération, quand le signifiant zéro rencontre l'élément extra-systématique favorable (élément qui est donc le catalyseur).

5. Il faut remarquer que dans Le neutre (Barthes, 1978), en particulier dans le chapitre sur l'ideosphère, Barthes conduit une exploration du néologisme qui semble juste s'arrêter devant l'invention du terme lotmanien de sémiosphère.

6. Cf. Gödel (1953). 
Chez Barthes, la reprise du terme catalyse marque un détachement progressif de l'héritage hjelmslevien, ce qui implique une reconceptualisation de la notion. Dans les Éléments de sémiologie, la catalyse n'est que la saturation syntaxique, relevant de l'ampleur du paradigme admis, après un élément déjà manifesté (Barthes, 1965, p. 681); de manière indépendante, après quelques pages, on trouve une synthèse des réflexions portant sur le signifiant zéro, synthèse qui cite la même référence à Henri Frei que chez Lotman. Nous sommes en 1965, la connexion entre le signifiant zéro et la catalyse n'est pas encore totalement articulée dans la pensée barthésienne. Il faut attendre l'Introduction à l'analyse structurale des récits, c'està-dire $1966^{7}$.

Si Greimas avait tendance à voir la catalyse comme une présupposition forte dans des schématismes narratifs, Barthes pensait, au contraire, à la catalyse comme un phénomène textuel réalisé, à savoir un ralentissement par rapport à la syntaxe des fonctions narratives cardinales ${ }^{8}$, ralentissement qui implique « des zones de sécurité, des repos, des luxes" (Barthes, 1966c, p. 841). La lecture plutôt idiosyncratique de la notion hjelmslevienne, en tant que «saturation syntaxique», va continuer à avoir un poids déterminant : Barthes parle de l'absence d'une absence, de la non-économie textuelle, du fait que le texte raisonne à l'envers de l'abstraction métalinguistique de la théorie; c'est pourquoi il veut ajouter toujours des interstices (ibid., p. 861).

La catalyse [écrit-il] réveille sans cesse la tension sémantique du discours. [Elle] dit sans cesse : il y a eu, il va y avoir du sens; la fonction constante de la catalyse est donc, en tout état de cause, une fonction phatique. (ibid., p. 841).

Mais bien évidemment «le pouvoir catalytique du récit a pour corollaire son pouvoir elliptique» (ibid., p. 842). Certes, au signifiant vide, Barthes commence à substituer le "plein", voire le "trop plein", d'où son intérêt pour Sade, où la phrase " est elle aussi un corps qu'il faut catalyser, en remplissant tous ses lieux premiers (sujet-verbe-complément) d'expansion, d'incises, de subordonnées, de déterminants » (Barthes, 1971, p. 814).

Pourquoi cette obsession barthésienne pour une catalyse déjà réalisée sur la surface textuelle et de manière si exacerbée? L'explication est bien présente dans Sade, Fourier, Loyola : bien qu'on puisse chercher à saturer tous les interstices possibles, le résultat est catastrophique, car cela ne produit que la révélation d'autres vides.

7. Par ailleurs, toujours dans Sign, Language, Culture, où Barthes a publié «La linguistique du discours ", on trouve quelques pages après, l'intervention d'Anna Wierzbicka (1965, p. 627) qui soulignait «The postulate of our marking out all the heterogeneous elements of the given text before starting the semantic (or grammatical) analysis is a matter of fact kindred to the postulate of catalysis (completing empirical text). Empirical texts are full of abbreviation or elliptical phrases [...] Now, the situation is similar with respect to heterogeneous "insertions" ".

8. «Les catalyses ne sont que des unités consécutives, [en revanche] les fonctions cardinales sont à la fois consécutives et conséquentes. » (Barthes, 1966c, p. 840). 
Par rapport aux catalyses manifestées comme pour saturer les enjeux communicatifs, la conclusion barthésienne est totalement antiphrastique : « rien ne permet (structurellement) de terminer une phrase» - dit Barthes (ibid., p. 814). Chaque récit de catalyse accomplie est le révélateur des vides, des signifiants-zéro qui traversent encore le plan de la manifestation textuelle. Les catalyses explicites ne sont que la tentative paradoxale de se cacher les vides qui affectent les jeux de langage, même si elles cherchent bien à saturer les potentialités combinatoires. Ainsi, les catalyses manifestées ne sont qu'un supplément qui déplace seulement la confrontation finale du texte avec son besoin d'éléments externes. L'irruption de l'élément externe dans un texte qui utilise la technique de l'atermoiement, de la prolifération d'éléments non-cardinaux, ne peut que provoquer une implosion de sa prétendue clôture.

La conclusion est qu'il ne faut pas attendre un texte elliptique pour voir l'émergence de la catalyse. Là où elle semble déjà satisfaite, on trouve seulement le plaisir fragile d'une saturation asymptotique qui enquête ainsi sur ses trous, ses vides et sur l'irruption tôt ou tard d'une étrangeté, d'une composante hétérogène. Chaque texte est alors plus ou moins peuplé de catalyses inachevées, résistantes, qui montrent comment le démêlage du tissu textuel dégage aussi des effets de sens au-delà des contraintes strictement immanentes.

\subsection{Le jeu libre et l'orientation à la variabilité}

Les conséquences théoriques de ce discours sont très importantes : la reconstruction du sens à travers la modélisation sémiotique d'une langue qui devient parole, selon un processus de déterminations successives, à partir de la même base sémantique et syntaxique, ne peut jamais rencontrer la signification vive d'un texte historique. De l'énoncé à l'énonciation, il y a un manque d'explication et l'abduction ne peut que procéder par l'interpolation d'autres médiations que celles qui ont été directement introjectées par le texte (cf. Parret, 1986, p. 152). Cela ne peut qu'attribuer un rôle critique fondamental à l'interprète. Si un objet culturel est toujours en train d'exhiber le fait qu'il s'adapte de manière solidaire ou contrastive à la scène sociale d'implémentation publique, l'interprète doit chercher les catalyses inachevées, les sutures possibles entre les vides; des vides qui ne sont pas des présuppositions internes à la même organisation structurale, mais des éléments manquants qui appellent d'autres structures et d'autres espaces culturels.

Lotman était profondément convaincu de l'hybridité des principes sémiotiques et de l'impossibilité de saturer la relation entre la déclinaison des contraintes des systèmes et le caractère historico-culturel du texte. Au parcours génératif de la signification, Lotman aurait pu opposer une suite de transitions et de passages entre un vide et l'autre. Cela n'implique pas une perte d'identification et de description possibles des phénomènes discursifs, mais montre immédiatement les tensions entre la pluralité des organisations internes (phonologie, grammaire, sémantique) et des éléments externes comme le mètre, qui peut sur-ordonner soit les phonèmes, 
soit les grammèmes, soit les sémantèmes (M. Lotman, 1974). En tout cas, il y a des relations libres, irréfléchies qui deviennent le catalyseur d'un sens non prévu.

La possibilité de choix à différents niveaux, l'intersection de différents types d'organisations et le «jeu» libre entre eux font partie du minimum de mécanismes culturels indispensables (Lotman, 1973, tr. fr. p. 190).

Selon Lotman, chaque culture est un " ensemble complexe et contradictoire " (Lotman, 1971b, tr. it. p. 72), et la difficulté de la théorie à suturer la relation entre la modélisation générative et les phénomènes textuels trouve une démonstration accablante dans le fait que l'auto-description d'une culture ne peut jamais éviter de passer par des degrés de déstructuration de la textualité produite (vu l'excès de grammaticalisation), des degrés d'idéalisation (avec un hiatus plus ou moins dramatique entre utopie et réalité), des degrés de fiction délibérée (la prolifération autoréférentielle des lois dépasse la rationalisation des pratiques) (ibid., p. 73).

Si la culture échappe à son autodescription complète, elle doit constater aussi l'impossibilité de coder et de diriger les fluctuations des systèmes dans le temps : le changement phonétique et la mode en sont une démonstration (Lotman, 1971a, tr. it. 62). La mode est un système "orienté à la variabilité » de sorte que toute normativité affirmée localement est déjà prête à laisser le terrain à d'autres propositions formelles (ibid., p. 63). Le vide explicatif est programmatique, ce qui montre que le dynamisme culturel est une intersection des catégories où il y a du "jeu", à savoir un espace de transition qu'on ne peut pas saturer, un espace où on assiste à l'« infini permutatoire du langage » - selon l'expression très idiolectale de Barthes (1970a, p. 659) déjà citée (voir $\$ 2.1)$.

\subsection{Du paradigme à l'environnement : l'étrangeté nécessaire}

Le "changement pur", en tant que facteur de renouvellement, témoigne du fait que les langages ne peuvent pas saturer l'espace de vie d'une culture. On reconnaît alors l'existence d'un environnement qui est au-delà de tous les espaces d'implémentation des objets culturels. Naturellement, dans la perspective lotmanienne, cet environnement est composé surtout par l'hétérogénéité des systèmes culturels à laquelle chaque point de vue doit faire face en fonction de sa position. L'impénétrabilité des autres systèmes montre une catalyse interprétative nécessaire qui ne peut que produire des informations, des valeurs d'innovations. L'hétérogénéité et l'alternativité des systèmes sont à la base des vides tout comme des effets "boule de neige », en exemplifiant des valeurs intraduisibles qui vont se traduire malgré tout, en favorisant paradoxalement un «changement accéléré des mécanismes informatifs en acte ${ }^{9}$. Ainsi, la catalyse devient aussi un facteur de métaobservation : signe des signes qui manquent, signe d'une implicitation au carré, où les interrogatifs de l'existence peuvent activer le "mouvement vers l'infini » qui émergeait de l'étude de l'espace artistique chez Gogol.

9. Lotman (1971a, p. 67). 
À la fin de son dernier livre en italien, Chercher la route, Lotman décrit la connaissance comme si elle était sous l'empire d'un rêve sans espoir : "l'idée se rejoint elle-même en toute hâte ", afin de boucler la boucle autodescriptive (Lotman, 1994, p. 106). Par contre, la culture est pleine d'asynchronismes, de processus décalés, de ralentissements et de moments de forte accélération, provoqués par la réception d'un élément externe qui remplit un interstice vide de la culture d'accueil. Le dialogue plurilinguistique est alors à la base du dynamisme des systèmes sociaux et l'importation/traduction a des effets explosifs, avec des retombées sur le futur et sur la mémoire aussi (ibid., p. 38). Pour Lotman, la traduction est le véritable élément catalyseur qui montre l'impact de l'espace extrasémiotique sur l'objet culturel (Lotman, 1985, p. 60). Paradoxalement, c'est à la périphérie d'une culture, là où les autodescriptions sont plus rares, qu'on trouve une accélération du changement (ibid., p. 64). À la périphérie, les formations sémiotiques sont plus fragmentaires et les fragments étrangers fonctionnent dit Lotman de manière explicite - comme des «catalyseurs ». L'interrogation prééminente chez Lotman devient alors la suivante : "Quelles sont les conditions et les situations qui expliquent pourquoi un texte étranger devient nécessaire? » (ibid., p. 116). Ces conditions ne sont pas la recherche d'une stabilité majeure, mais au contraire l'augmentation de l'indétermination interne (ibid., p. 128).

Dans un système avec une grande indétermination interne, entre de l'extérieur un texte qui, vu qu'il est un texte et non seulement un "sens nu " (cf. Zolkovski et Shcheglov), gagne lui-même une indétermination interne, en se présentant moins comme une réalisation concrète d'un langage que comme une construction polyglotte susceptible d'une série d'interprétations [polylinguistiques, conflictuelles et innovatrices]. (Lotman, 1985, p. 128).

La catalyse est alors une « fenêtre dans la strate sémiotique » qui permet finalement de « "jouer" avec l'espace qui est externe à cette dernière, tantôt l'absorbant, tantôt y rejetant les éléments utilisés qui ont perdu leur activité sémiotique » (Lotman, 1993; tr. fr. p. $48^{10}$ ).

\subsection{Questions de rythme: la mode et le jeu}

La mode et le jeu semblent se proposer comme les domaines d'exemplification d'un dynamisme de la culture où la phase statique, synchronique, est une pure fiction qui aide l'autodescription des systèmes. À ce propos, «la mode apporte du dynamisme dans les sphères de la vie qui semblent immobiles " (Lotman, 1993, tr. fr. p. 112). Le caractère capricieux et immotivé de la mode "devient une sorte de métronome de l'évolution culturelle » qui rythme la «transformation de l'insignifiant en signifiant» (ibid., p. 112-113).

La catalyse peut être saisie comme ponctuelle (explosive), mais elle se cache aussi dans des configurations itératives (rythmiques), en relançant ainsi des

10. Trad. modifiée. 
mouvements agogiques très différenciés, à cause des accélérations et des ralentissements asymétriques des transformations. "L'espace sémiotique est rempli par des fragments de différentes structures » qui se déplacent, se heurtent, se restaurent brusquement (ibid., tr. fr. p. 151). Mais la mode ne traite pas seulement les fragments internes à travers une sorte de bricolage continu ; elle se pose aussi comme la "forme d'irruption» d'une langue extérieure qui, avec son rythme, "s'insère dans la dynamique de la culture principale ».

Dans cette idée que la mode " est destinée à être le métronome et le catalyseur du développement culturel ", la voix de Lotman semble se superposer totalement à celle de Barthes. La fascination pure exercée par un espace extérieur se traduit dans l'accueil de sa prosodie, de ses phénomènes de variation et des opportunités de changement données à la langue d'arrivée.

Il faut surtout prendre au sérieux le rôle de la catalyse, car l'explosion opère sur un chaînon précis d'une création, d'un texte, d'une formation discursive. La catalyse est donc la fausse synonymie qui s'affirme là où une forme paradigmatique substitutive ne peut plus être garantie par la culture d'origine (cf. Lotman, 1993, tr. fr. p. 174). À la centralité d'un fond de structures générales, on substitue un principe de formes nomades, en quête d'assimilation et d'assomption, selon une structuration dynamique et locale. Selon Lotman, «l'art représente non pas "un jeu sans règles", mais un jeu dont il faut fixer les règles au cours du jeu » (Lotman, 1970, tr. fr. 401). C’est pourquoi Lotman n'a jamais proposé ou accepté un modèle structural génératif (ibid., p. 402).

Dans "La thèse sur l'art en tant que système de modélisation secondaire ", Lotman montre que le jeu est à la base de l'autotélie de l'art, de sa justification autonome, vu son caractère fictionnel et son émancipation par rapport au réel : «le jeu est l'indispensable structure émotive pour toute activité pratique envisageable " (Lotman, 1967, p. 8), une structure de conciliation, transversale, qui implique en même temps introjection et suspension, convention et expérimentation. Le jeu " cherche à simuler le hasard, la détermination imparfaite » et il s'introduit comme un fond actif par rapport au principe de la langue (ibid., p. 11).

La traduction de l'intraduisible, passage sans aucun doute crucial dans l'explosion lotmanienne, trouve une correspondance dans l'" imitation de l'inimitable " qui caractérise la vision barthésienne de la mode (Barthes, 1966b, p. 887). Or, le "jeu", la possibilité de dépasser la différence radicale, est d'accepter une proposition rythmique où la forme étrangère n'est que le catalyseur d'une accélération dans un certain domaine de la culture d'accueil. La mode est alors un facteur d'asymétrisation et d'asynchronisation des dynamiques sociales. Les structures rythmiques n'ont pas de problème de justification, de validation; leur diffusion capricieuse profite de l'impertinence de toute rationalisation.

L'intrusion du détail extérieur est favorisé par la présence d'un interstice, d'un signifiant zéro dans la culture d'accueil; ce signifiant zéro est d'autant plus visible ("nu") que la culture d'accueil est statique, dépourvue d'un véritable rythme. Mais 
la présence massive des ellipses peut bien être paralysante, dissuasive. L'intrusion rythmique est alors catalytique car elle cache un remplissage sur le plan du contenu imprévu. Bref, il nous semble que notre réflexion commune sur les relations entre Barthes et Lotman a bien dépassé l'artifice rhétorique ou la reconstruction historique érudite, en montrant des intersections réelles et productives, au point qu'on pourrait trouver, à travers la synthèse des contributions, une base fiable pour une formalisation de la catalyse.

\subsection{L'ellipse et le trop plein}

Il est intéressant de préserver la notion de catalyse car elle montre que l'assomption de la forme étrangère est révélatrice d'un chaînon manquant dans l'autodescription de la culture d'accueil, ce qui veut dire que cette dernière rencontre l'étranger, voire l'“aliène", comme une partie implicite d'elle-même.

Sur le plan historique, Barthes remarque que la démocratisation des formes de vie, avec des styles disponibles finalement pour tous, oblige à prendre au sérieux le détail distinctif (Barthes, 1962, p. 28). Ce détail peut avoir l'apparence du rien, du « je ne sais quoi ». L'intrusion de la distinction est alors révélatrice d'un vide implicite qui peut être comblé avec un détail encore indéterminé. Le procédé chimique qui active le changement des productions sémiotiques et, en particulier, la formule qu'institue le soi comme un autre, sont prêts.

Il faut se rappeler que pour Barthes les aspects les plus intéressants sont, d'une part le "trop plein" de la catalyse manifestée dans l'énoncé, jusqu'à la prolifération baroque, d'autre part l'ellipse : «figure mal connue, trouble en ceci qu'elle représente l'effroyable liberté du langage, qui est en quelque sorte sans mesure obligée » (Barthes, 1975, p. 657). L'ellipse n'est que la catalyse manquante sur le plan de l'énoncé, mais l'aspect le plus remarquable est que ce manque silencieux peut être associé à une absence paradigmatique : l'ellipse devient alors un véritable symptôme de la faille du système linguistique, la révélation de sa vulnérable neutralité par rapport à l'avènement d'un élément étranger. L'ellipse devient ainsi un véritable enjeu culturel quand il n'y a pas de substitutions connotées, quand il y a seulement le vide, quand l'initiative de l'énonciateur est neutralisée. Y a-t-il encore la possibilité d'un « déclic paradigmatique » dans l'ouverture du système, l'explosion peut-elle être une catalyse ponctuelle qui comble le vide dans le tissage textuel?

\section{Conclusions. La vie des systèmes culturels}

On peut finalement tirer des conclusions en se demandant comment Lotman et Barthes ont pu combiner une vocation critique de l'analyse sémiologique avec une focalisation sur l'indétermination et l'impersonnalité du changement culturel. Le dandy est le tout dernier héros qui cherche à travailler de manière autonome sur les détails distinctifs, car « la mode a exterminé toute singularité pensée du vêtement ; 
[elle a inoculé] un peu de dandysme à tout le vêtement contemporain " (Barthes, 1962, p. 30).

$\mathrm{Au}$ fond, Barthes et Lotman ont travaillé sur un modèle de transmission culturelle qui ne serait pas liée à la puissance unilatérale des codes, ni à un modèle épidémiologique. Ils ont dessiné chacun avec son propre horizon théorique, une vie des systèmes culturels, une vie infléchie par des facteurs indéterminés, par des jeux de forme qui sont la garantie aussi de la dynamisation continue de la sémiose sociale. La vie des systèmes accompagne les protagonistes de l'histoire, mais en démythisant leurs actions, ce qui ne permet plus de les voir comme des héros. Le choix d'un destin tout comme l'auto-observation de sa propre conduite sont, sur le plan individuel et collectif, une tension imperfective, où le studium (la concentration volontaire) doit s'articuler avec le punctum de l'explosion formelle imprévue. Bref, la théorie même ne peut plus avoir de prétentions "hérö̈ques" : la nécessité de faire face à une hétérogénéité qui empêche un plan de description unifiant a motivé, peut-être, la renonciation progressive de Barthes et Lotman à la formalisation et, de plus en plus aussi, à la schématisation.

La lecture croisée de Barthes et Lotman démontre qu'ils n'ont pas cherché à trouver une aptitude descriptive cachée dans les pratiques et dans les textes produits; au contraire, les formes de vie et les discours étudiés ont été assumés comme une posture excentrique que le regard du sémiologue doit apprendre à assumer. La vie culturelle n'est qu'une série d'anamorphoses qui rêvent de s'imposer comme une "perspective centrale". Le rôle éthique de la théorie est la critique de cette ambition, non la réalisation de cette dernière. C'est pourquoi Barthes accepte de plus en plus son implication dans l'objet, vers une sorte de sémiologie expérientielle; c'est pourquoi les essais de Lotman donnent la parole, avec sollicitude et finesse critique, aux littéraires et aux artistes ou encore aux ruses de la pensée populaire.

$\mathrm{Au}$ fond, un discours sur la culture, une autodescription de la culture comme le métadiscours sémiotique, ne peut pas éviter de reproduire en soi une autocatalyse. Comme le disait Lotman au début du livre Universe of the Mind:

L’idée que les structures sémiotiques "pensantes" nécessitent une impulsion initiale d'une autre structure de pensée et que ses mécanismes de génération textuelle demandent un texte extérieur afin de les mettre en mouvement, nous rappelle, d'une part, le rôle des réactions autocatalytiques, à savoir les réactions où la réalisation d'un produit final (ou l'accélération d'un processus chimique) doit être déjà présente, dans une certaine mesure, au début de la réaction même; d'autre part, cette question trouve un parallélisme dans le problème encore insoluble qui concerne le début de la culture et le début de la vie. (Lotman, 1990, p. $\left.3^{11}\right)$.

11. Traduction personnelle. Voici le texte-source: "The idea that "thinking" semiotic structures need an initial impulse from another thinking structure and that text-generating mechanisms need a text from outside to set them going reminds us on the one hand of so called autocatalytic reactions, that is those reactions where, in order to obtain the final product (or to hasten a chemical process), the final result has to be already present in some quantity at the beginning of the reaction. And on 
Au-delà de l'ambition de prendre en charge l'origine de la culture et des langues, on peut bien profiter de la catalyse comme détecteur sémiotique de la dynamisation des systèmes, ce qui peut conjuguer une vision écologique de la culture avec une perspective indiciaire ou archéologique de l'analyse du discours. $\mathrm{Au}$ fond, nous ne voulons pas défendre l'idée que Lotman et Barthes auraient partagé la même conception de catalyse, malgré plusieurs points de contact. Ce que nous avons proposé est une sorte d'application récursive du principe catalytique à travers deux conceptualisations différentes mais complémentaires de ce procès, jusqu'au point d'envisager une théorisation syncrétique meilleure du procès infiniment productif de la culture. La convergence entre Barthes et Lotman est alors parfaitement explosive.

\section{Références bibliographiques ${ }^{12}$}

Barthes, Roland (1953), Le Degré zéro de l'écriture, Paris, Seuil; nouv. éd. Euvres complètes, vol. I, Paris, Seuil, 2002, pp. 169-226.

- (1957), Mythologies, Paris, Seuil; nouv. éd. Euvres complètes, vol. I, Paris, Seuil, 2002, pp. 671-870.

- (1960a) «Le bleu est à la mode cette année », Revue française de sociologie, vol. I, n. 2; nouv. éd. CEuvres complètes, vol. I, Paris, Seuil, 2002, pp. 1025-1038.

- (1960b) « Les unités "traumatiques" au cinéma » (en collaboration avec G. Cohen Séat), Revue International de filmologie, X, n. 34; nouv. éd. Euvres complètes, vol. I, Paris, Seuil, 2002, pp. 1047-1056.

- (1962), « Le dandysme et la mode », United States Lines Paris Review, juillet 1962; nouv. éd. CEuvres complètes, vol. II, Paris, Seuil, 2002, pp. 27-31.

- (1965), Éléments de sémiologie, Paris, Denoël/Gonthier; nouv. éd. Euvres complètes, vol. II, Paris, Seuil, 2002, pp. 631-704.

- (1966a), Critique et vérité, Paris, Seuil; nouv. éd. CEuvres complètes, vol. II, Paris, Seuil, 2002, pp. 757-801.

- (1966b), «La mode et les sciences humaines », Échanges, Assomption; nouv. éd. CEuvres complètes, vol. II, Paris, Seuil, 2002, pp. 887-892.

- (1966c), "L'Introduction à l'analyse structurale des récits », Communications, no 8 , novembre 1966; nouv. éd. CEuvres complètes, vol. II, Paris, Seuil, 2002, pp. 828-865.

the other hand, this question finds a parallel in the as yet unsolved problem of the "beginning" of culture and the "beginning" of life. » (Lotman, 1990, p. 3).

12. L'article fait référence à des versions italiennes ou anglaises des textes de Lotman s'il n'y a pas une traduction disponible en français. L'auteur a traduit en français les versions étrangères approuvées par Lotman afin de faciliter la lecture. Le texte d'origine, si étendu, est en note de bas de page. 
- (1966d), "La linguistique du discours », in A. Greimas et al. (éds), Sign, Language, Culture, The Hague, Mouton, 1970, pp. 580-584; nouv. éd. Euvres complètes, vol. III, Paris, Seuil, 2002, pp. 611-616.

- (1970a), "Sur “S / Z” et “L’empire des signes" » (avec R. Bellour), Les Lettres françaises, 20 mai 1970; nouv. éd. Euvres complètes, vol. III, Paris, Seuil, 2002, pp. 655-670.

- (1970b), S/Z, Paris, Seuil ; nouv. éd. Euvres complètes, vol. III, Paris, Seuil, 2002, pp. 119346.

- (1970c), «L'ancienne rhétorique. Aide-mémoire», Communications, $\mathrm{n}^{\circ}$ 16, décembre 1970 ; nouv. éd. Euvres complètes, vol. III, Paris, Seuil, 2002, pp. 527-601.

- (1970d), «Critique et autocritique»(entretien avec André Bourin), Les Nouvelles littéraires, 5 mars 1970; nouv. éd. Euvres complètes, vol. III, Paris, Seuil, 2002, pp. 635-648.

- (1971), Sade, Fourier, Loyola, Paris, Seuil; nouv. éd. Euvres complètes, vol. III, Paris, Seuil, 2002, pp. 699-868.

- (1972), «Sémiologie et médecine ", in R. BAstide (éd.), Les Sciences de la folie, The Hague, Mouton; nouv. éd. Euvres complètes, vol. IV, Paris, Seuil, 2002, pp. 174-183.

- (1973), Le Plaisir du texte, Paris, Seuil; nouv. éd. Euvres complètes, vol. IV, Paris, Seuil, 2002, pp. 217-264.

- (1975), Roland Barthes par Roland Barthes, Paris, Seuil; nouv. éd. Euvres complètes, vol. IV, Paris, Seuil, 2002, pp. 575-771.

- (1978), Le Neutre. Cours au Collège de France (1977-1978), Paris, Seuil, 2002.

Frei, Henri (1950), «Zéro, vide et intermittent », Zeitschrift für Phonetik und allgemeine Sprachwissenschaft, Academie Verlag, n. 1, 6 (3-4), pp. 161-191.

GöDEL, Robert (1953), "La question des signes zéro », Cahiers Ferdinand de Saussure, n. 11, pp. 31-41.

Goodman, Nelson (1968), Languages of Art. An Approach to a Theory of Symbols, London, Bobbs-Merril; tr. fr. Les Langages de l'art. Une approche de la théorie des symboles, Nîmes, J. Chambon, 1990.

Greimas, Algirdas J. \& Courtés, Joseph (1979), Sémiotique. Dictionnaire raisonné de la théorie du langage, Paris, Hachette.

Hjelmslev, Louis (1943), Omkring Sprogteoriens Grundloggelse, København, Munksgaard; tr. fr. Prolégomènes à une théorie du langage, Paris, Minuit, 1971.

Likhatchov (Likaciev), Dmitri Sergeïevitch (1962), Tekstologija, na materiale russkoj literatury, X-XVII vv, Moscou et Léningrad, AN SSSR; nouv. éd. St. Petersburg, Aleteyya, 2001.

Lotman, Youri M. (1964), «Tekstovye i vnetekstovye struktury», chap. III de "Lektsii po strouktoural'noï poetiké», Troudy po znakovym sistemam, Tartu, Outch. zap. Tart. ouniversiteta, 1964; tr. it. «Il problema del testo », in Neergard (éd.), Teorie contemporanee della traduzione, Milano, Bompiani, 1995. 
- (1967), "Tezisy k probleme "Iskusstvo v rjadu modelirujuščich sistem" "; tr. it. "La tesi sull'arte come sistema modellizzante secondario », in Lotman \& OUSPENSKi (éds), Semiotica e cultura, Napoli, Ricciardi Editore, 1975, pp. 1-27.

- (1970), Struktura chudozestvennogo teksta, Moskva, Iskusstvo; tr. fr. La Structure du texte artistique, Paris, Gallimard, 1973.

- (1971a), "O semiotičevskom mechenizme kul'tury», Trudy po znakovym sistemam, fasc. 284, 5, Tartu, pp. 114-66; tr. it. "Il meccanismo semiotico della cultura », in Y. Lotman \& B. Ouspenski, Tipologia della cultura, Milano, Bompiani, 1973, pp. 3968.

- (1971b), «Problema “obučenija kul'ture" kak ee tipologičeskaja charakteristika ", Trudy po znakovym sistemam, fasc. 284, 5, Tartu, pp. 167-76; tr. it. " La cultura e il suo "insegnamento" come caratteristica tipologica ", in Y. Lotman \& B. OUsPenski, Tipologia della cultura, Milano, Bompiani, 1973, pp. 69-81.

- (1973), « Scena i živopis' kak kodirujuščie ustrojstva kul'turnogo povedenija čeloveka načala XIX stoletija », in Y. Lotman, Stat'i po tipologi kul'tury, 2, Tartu, pp. 74-89; tr. fr. in Y. Lotman \& B. Ouspenski, Sémiotique de la culture russe, Lausanne, L’Âge de l'Homme, pp. 179-191.

- (1975a), «Dekabrist v povsednevnojz $z^{v}$ izni », in BAZAnov \& VAcuro (éds), Literaturnoe nasledie dekabristov, Leningrad, Nauka; tr. fr. "Le décembriste dans la vie quotidienne ", in Lotman \& Ouspenski, Sémiotique de la culture russe, Lausanne, L’Âge de l'Homme, 1990, pp. 89-139.

- (1975b), «Khlestakov», Učënie zapiski, 369, TRSF XXVI, Tartu; tr. fr. "À propos de Khlestakov", in Lotman \& Ouspenski, Sémiotique de la culture russe, Lausanne, L'Âge de l'Homme, 1990, pp. 192-226.

- (1985), La semiosfera, Venezia, Marsilio.

- (1990), Universe of the Mind, London \& New York, I. B. Tauris \& Co; tr. fr. part. La Sémiosphère, Limoges, PULIM, 1999.

- (1993), Kultura i Vzryv, Moskva, Gnozis; tr. fr. L'Explosion et la Culture, Limoges, PULIM, 2004.

- (1994), Cercare la strada, Venezia, Marsilio.

Lotman, Youri M. \& Ouspenski, Boris (1973), Tipologia della cultura, Milano, Bompiani.

- (1975), Semiotica e cultura, Napoli, Ricciardi.

- (1990), Sémiotique de la culture russe, Lausanne, L’Age de l'Homme.

Lotman, Youri M. \& Ouspenski, Boris (éds, 1973), Ricerche semiotiche, Torino, Einaudi.

- (éds, 1976), Travaux sur les systèmes de signes, Bruxelles, Éditions Complexe.

Lotman, Mihhail Y. (1974), «O vzaimodeystvii yestestvennogo yazyka i metriki v mekhanizme stikha», ТГУ, vol. 1 (5), Тарту, pp. 182-186; tr. fr. "L'interrelation de la langue naturelle et du mètre dans le mécanisme du vers ", in Y. Lotman \& B. Ouspensky (éds), Travaux sur les systèmes des signes, Bruxelles, Éditions Complexe, 1976. 
Parret, Herman (1986), Les Passions, Liège, Mardaga.

Wierzbicka, Anna (1965), « Descriptions or quotations », in A. Greimas et al. (éds), Sign, Language, Culture, The Hague, Mouton, 1970, pp. 627-644.

Zenkine, Serge (2010), «La sémiotique de l'image visuelle : Roland Barthes et Iouri Lotman ", in M. Costantini (éd.), Glissements, décentrements, déplacement. Pour un dialogue sémiotique franco-russe, Texte électronique, Université de Paris VIII, pp. 8491. 